\title{
Analysys of the impact of disturbance on the arteriovenous fistula state classification
}

\author{
Marcin Grochowina \\ University of Rzeszów \\ al. Rejtana 16, 35-310 Rzeszów, Poland \\ Email: gromar@ur.edu.pl
}

\author{
Lucyna Leniowska \\ University of Rzeszów \\ al. Rejtana 16, 35-310 Rzeszów, Poland \\ Email: lleniow@ur.edu.pl
}

\begin{abstract}
The quality of data sets used in the classification process has a significant impact on the outcome. The noise contained in the input data depending on the nature and intensity may have a different effect on the classification result. This paper presents the results of research on the quality and reliability of arterio-venous fistula classification based on the signal recorded under controlled disturbance conditions and in the model of artificial disturbations.

Typical environmental noise that may occur when the acoustic signal produced by the fistula was recorded and it is used as a disturbance. Its influence on the features extraction process and on the result of the fistula assessment was determined. Finally, a relationship between the intensity of the disturbances and the degree of shifting of the classification result to the pathological state of the fistula was demonstrated
\end{abstract}

\section{INTRODUCTION}

$\mathbf{T}$ HE problem of disturbations contained in the data sets in classification process is a well-known phenomenon. Each data recording is affected by external factors, and disturbations results in classification errors [7].

As it is usually the case in medical context, even slight errors might lead to grave consequences. Thus, the quality in decision support is of particularly high importance. Diagnosis based on deformed data can carry dangerous implications for the patient. Therefore, in the area of medical diagnostics, classifiers are built in such a way that, in case of uncertainty, they indicate a worse condition than it is in real. Such diagnosis can be then verified by other methods. Too optimistic diagnosis could lead to oversight threatening life and health symptoms.

This paper presents the testing results of sensitivity of arteriovenous fistula state classification for disturbations. Because our decision support system is based on the analysis of the acoustic signal produced by the blood flowing through the fistula, it is sensitive to sounds coming from the environment [1]. The level of ambient noise in locations like medical rooms or a private apartments is far from ideal, hence, we have to ascertain the system performance under such conditions.

As part of the study, a fistula acoustic signal was recorded in the anechoic chamber (in silence). Then, the artificially mixed disturbances were added into the signals previously recorded. In case of some patients the signal has also been registered under specified distortion conditions with known intensity. The data obtained was analyzed for the effect of disturbances on the fistula state estimation error.

\section{MATERIALS AND METHODS}

The research material was registered in the dialysis station of the Regional Clinical Hospital No.2 in Rzeszów. Collected sounds of radio-cephalic and brachio-cephalic fistulas were recorded from 38 patients. Registration was performed using specially developed recording head [4].

The teaching set was based on records of 31 patients. A total of 2645 points were obtained in the feature space, with each point mapping the signal fragment analysis for a single heartbeat.

The data from remaining 7 patients was used to build a test set uncorrelated with the training set. The test set contained a total of 10561 points in the feature space.

The feature space, originally 23 dimensional, was reduced to 7 dimensions by selection procedure [3]. Features taken into consideration in the classification process contain information about the amount of power contained in the frequency spectrum at intervals of the tertian center frequencies 63, 80, 100, $315,400,500$ and $630 \mathrm{~Hz}$.

The state of the fistula was assigned to one of the six classes named $\boldsymbol{a}-\boldsymbol{f}$, where class $\boldsymbol{a}$ was the best, and the class $\boldsymbol{f}$ was the worst condition.

The experiment was performed using Weka 3.7.13 package[13] using the k-NN classifier with distance weighted voting and $k=10$ [2].

The recording process under natural conditions which takes into account the influence of external disturbance is shown in Fig.1.

However, two important issues emerged during the preparation of the research material:

1) natural sources of disturbances are not characterized by a single and fixed level of sound intensity,

2) there is no possibility of making recordings with all patients under controlled conditions of disturbance in the anechoic chamber.

Due to the inability to perform measurements under controlled conditions of a given level of disturbance, an artificial model of disturbance environment was used. For this purpose 


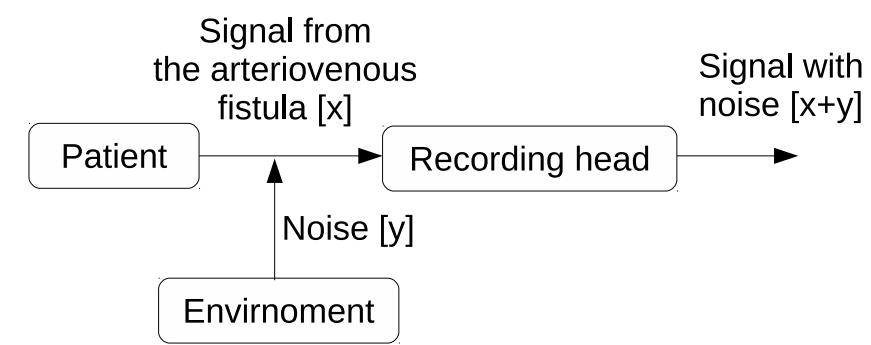

Fig. 1: The recording process under natural conditions

4 different sources were considered as potential disturbance signals (Tab.I):

- Urban noise - registered near the intersection of two busy streets,

- TV movie - sounds coming from a TV set that displays a movie,

- Conversation - lively discussion led by 3 people,

- Rock music - loudly played in the neighboring room hard rock music.

In addition, white noise as the fifth, control interfering signal was generated using software Audacity [14]. Registered and generated interfering signals were then used to create an artificial model of the noisy envirnoment.

TABLE I: Interference signals and their intensity

\begin{tabular}{l|c} 
Type of disturbance & Sound intensity during recording \\
\hline Urban noise & $70 \mathrm{~dB}$ \\
TV movie & $65 \mathrm{~dB}$ \\
Conversation & $60 \mathrm{~dB}$ \\
Rock music & $70 \mathrm{~dB}$
\end{tabular}

The impossibility of involving patients for multiple recording the fistulas sound under defined conditions was resolved by artificially mixing disturbances into the signals previously recorded in the dialysis station (Fig.2).

\section{Signal from}

the arteriovenous

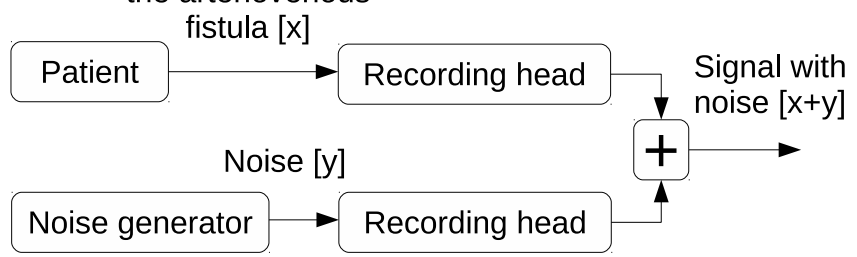

Fig. 2: The recording process with artificial disturbantion

For this purpose, two series of registrations were performed in the anechoic chamber, during which were recorded:

1) single patient fistula signal — during each recording, a previously recorded interfering signal with specific sound values of $55,60,65$ and $70 \mathrm{~dB}$ was played,

2) interfering signal - the recording header was applied to the patient's body at a distance from the fistula, and at the same time as the previous point, interfering sounds were played at the specified intensity.

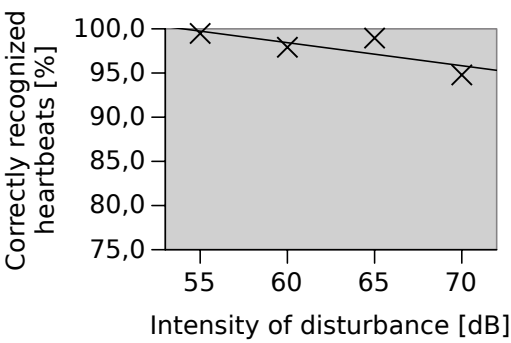

(a) White noise

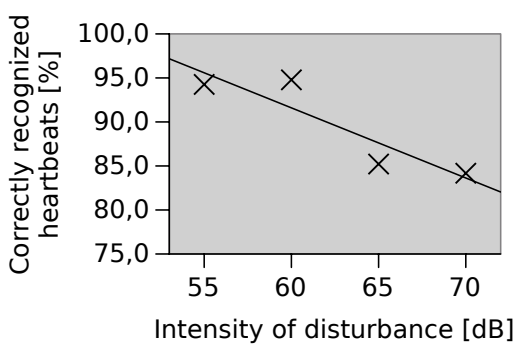

(b) Urban noise

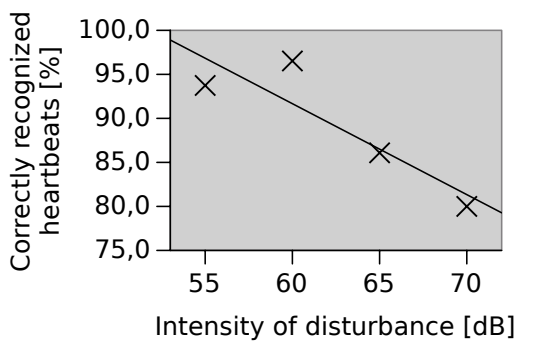

(c) TV movie

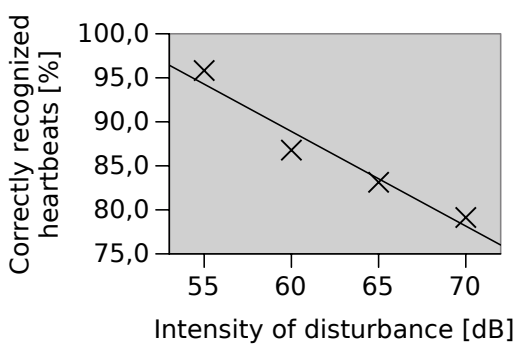

(d) Conversation

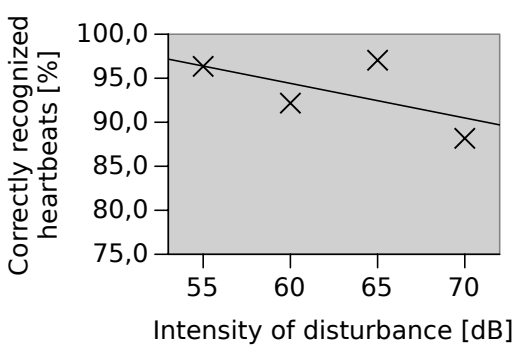

(e) Rock music

Fig. 3: Percentage of correctly recognized heartbeats in function of intensity of disturbance 
The volume value in both cases was measured with the Digital Sound Level Meter AZ8921 near the recording head [15].

Signals from patients registered in the dialysis station were added to the anechoic chamber recordings in Audacity. Each of the registered and artificially obtained signals was subjected to a classification process and the results were analyzed.

\section{RESULTS AND DISCUSSION}

A. The effect of disturbances on the operation of the feature extraction algorithm

First, after the registration of the research material, the effects of disturbances on the operation of the extraction algorithm were investigated [3].

For this purpose, the extraction algorithm was used on interrupted samples and on interfering samples. The disturbance was introduced into the signal in the artificial noising process.

The performance and robustness the feature extraction algorithm has no direct bearing on the quality of classification, however, increase the level of disturbance in a signal translates into a reduction of the number of points in feature space extracted from this signal.

The Tab.II shows the percentage of correctly extracted parts of signal corresponding to a single heartbeat. The number of heartbeats identified in the clean signal was assumed to be the baseline value.

TABLE II: Percentage of correctly recognized heartbeats

\begin{tabular}{c|ccccc}
$\begin{array}{c}\text { Intensity of } \\
\text { disturbance } \\
{[\mathrm{dB}]}\end{array}$ & White & Urban & TV & Conversation & Rock \\
noise & noise & movie & & music \\
\hline 55 & 99,5 & 94,3 & 93,7 & 95,8 & 96,3 \\
60 & 97,9 & 94,8 & 96,5 & 86,8 & 92,2 \\
65 & 99,0 & 85,2 & 86,1 & 83,1 & 97,0 \\
70 & 94,8 & 84,2 & 80,0 & 79,1 & 88,2
\end{tabular}

The data were presented graphically on Fig. 3. The charts were supplemented by trend lines.

In each of the cases studied, the increase in the intensity of the disturbances caused a decrease in the number of correctly interpreted heart rhythms.

The biggest impact on the number of correctly identified heartbeats had disturbances including sudden changes in sound intensity. Especially harmful were the conversation and containing a lot of dialogues television transmission of the TV movie.

White noise had the smallest effect on the extraction result. Because of its nature, it did not introduce sudden changes in sound intensity into the test signal, so it did not interfere with the process of detecting the extremes that indicate heartbeats.

Interestingly, rock music, as well as white noise, had little effect on the quality of the feature extraction algorithm, and apparently noise that is monotonous and devoid of sudden changes reduced the quality of extraction to $85 \%$.

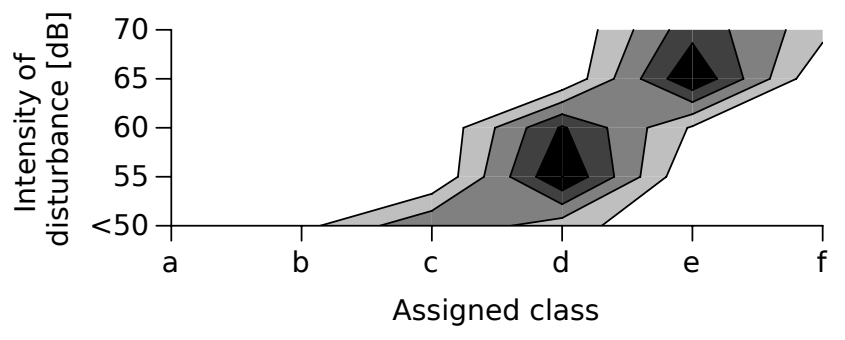

(a) White noise

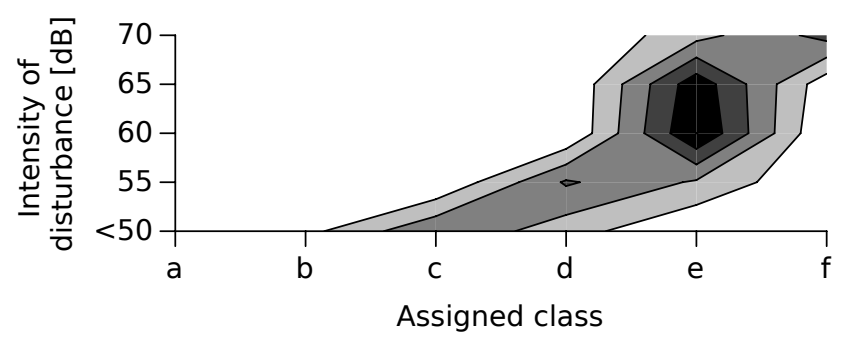

(b) Urban noise

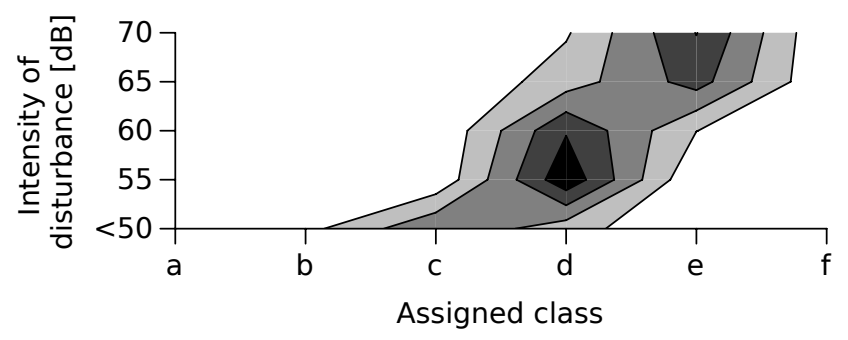

(c) TV movie

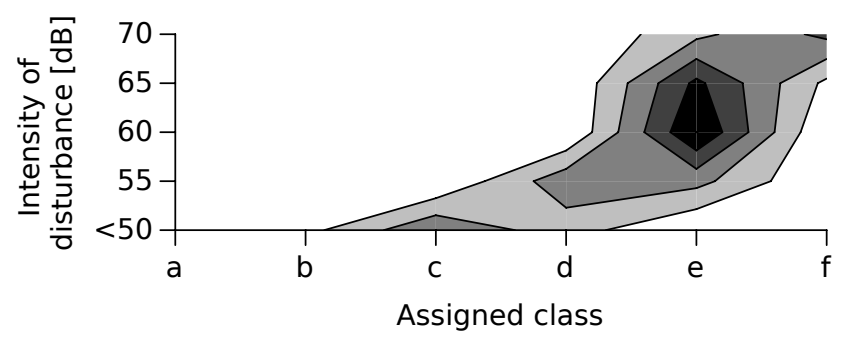

(d) Conversation

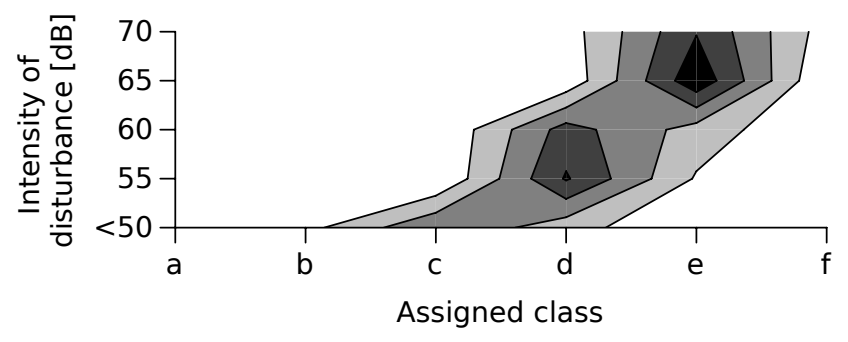

(e) Rock music

Fig. 4: Offset of the result of classification with increasing the disturbance intensity 


\section{B. Case study}

The next step was the analysis of a single case of a patient who agreed to register a fistula signal under controlled acoustic conditions of the anechoic chamber. During the registration of the fistula signal, the noise of a known intensity was played.

All the recorded signals were classified by the classifier built on the basis of 31 patients recorded with no disturbances. The results are shown in the Tab.III.

The first row of the table described "none" contains the result of analysis of 90 seconds of recording done in total silence. The results indicate that the condition of the examined patient's fistula was classified in class c. Leakage of results to neighboring classes $b$ and $d$ is the result of statistical uncertainty of classification and is a normal thing in the discussed problem of analysis of arteriovenous fistula state estimation.

TABLE III: Case 103 - complete result

\begin{tabular}{|c|c|c|c|c|c|c|c|}
\hline \multirow{2}{*}{$\begin{array}{l}\text { Type of } \\
\text { disturbance }\end{array}$} & \multirow{2}{*}{$\begin{array}{l}\text { intensity } \\
{[\mathrm{dB}]}\end{array}$} & \multicolumn{6}{|c|}{ Recognized class } \\
\hline & & $\mathrm{a}$ & $\mathrm{b}$ & $\mathrm{c}$ & $\mathrm{d}$ & $\mathrm{e}$ & $\mathrm{f}$ \\
\hline \multirow[t]{2}{*}{ none } & 0 & 0 & 11 & 46 & 23 & 0 & 0 \\
\hline & 55 & 0 & 0 & 0 & 80 & 0 & 0 \\
\hline White & 60 & 0 & 0 & 0 & 66 & 14 & 0 \\
\hline \multirow[t]{3}{*}{ noise } & 65 & 0 & 0 & 0 & 1 & 76 & 0 \\
\hline & 70 & 0 & 0 & 0 & 0 & 54 & 20 \\
\hline & 55 & 0 & 0 & 0 & 47 & 28 & 0 \\
\hline Urban & 60 & 0 & 0 & 0 & 0 & 74 & 0 \\
\hline \multirow[t]{3}{*}{ noise } & 65 & 0 & 0 & 0 & 0 & 65 & 5 \\
\hline & 70 & 0 & 0 & 0 & 0 & 21 & 43 \\
\hline & 55 & 0 & 0 & 3 & 66 & 1 & 0 \\
\hline TV & 60 & 0 & 0 & 1 & 58 & 15 & 0 \\
\hline \multirow[t]{3}{*}{ movie } & 65 & 0 & 0 & 0 & 19 & 43 & 1 \\
\hline & 70 & 0 & 0 & 0 & 11 & 50 & 1 \\
\hline & 55 & 0 & 0 & 0 & 40 & 35 & 0 \\
\hline \multirow[t]{3}{*}{ Conversation } & 60 & 0 & 0 & 0 & 0 & 76 & 0 \\
\hline & 65 & 0 & 0 & 0 & 0 & 61 & 11 \\
\hline & 70 & 0 & 0 & 0 & 0 & 23 & 43 \\
\hline \multirow{4}{*}{$\begin{array}{l}\text { Rock } \\
\text { music }\end{array}$} & 55 & 0 & 0 & 0 & 64 & 14 & 0 \\
\hline & 60 & 0 & 0 & 0 & 48 & 22 & 0 \\
\hline & 65 & 0 & 0 & 0 & 4 & 71 & 0 \\
\hline & 70 & 0 & 0 & 0 & 7 & 52 & 7 \\
\hline
\end{tabular}

The data in the table shows that even low intensity disturbances cause drastic changes in the assessment of the condition of the fistula. Each interfering signal tested has shifted the results of the classification towards the class indicating a condition that is worse than actual, with the amount of displacement increasing with the intensity of the disturbance.

Graphical visualization of results from the Tab.III was presented in Fig.4.

Areas marked with a darker color indicate the focus of classification hits on a given class. Gray areas indicate blurring results between classes.

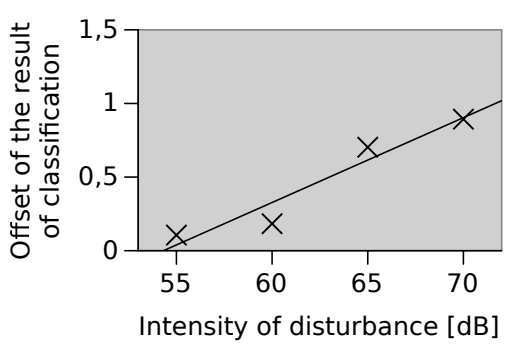

(a) White noise

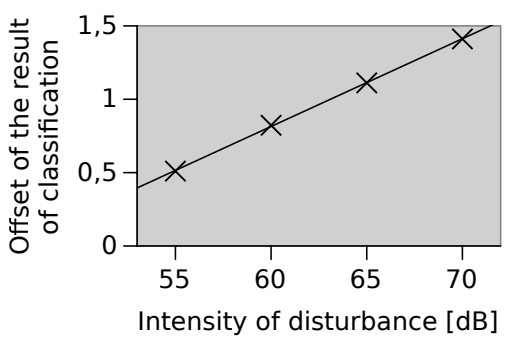

(b) Urban noise

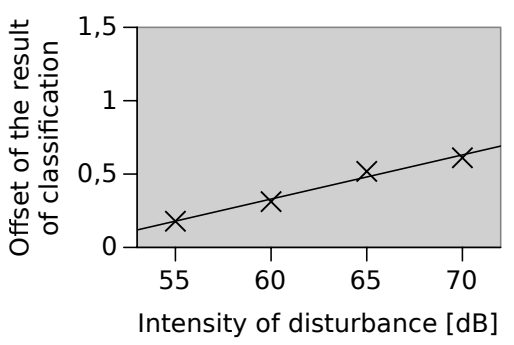

(c) TV movie

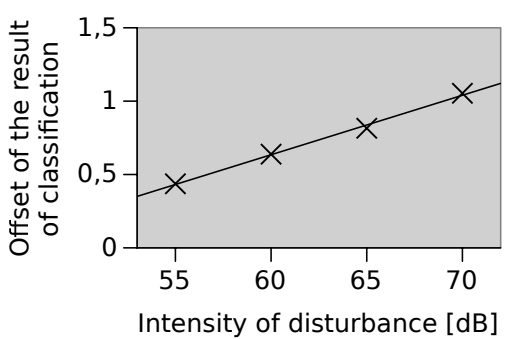

(d) Conversation

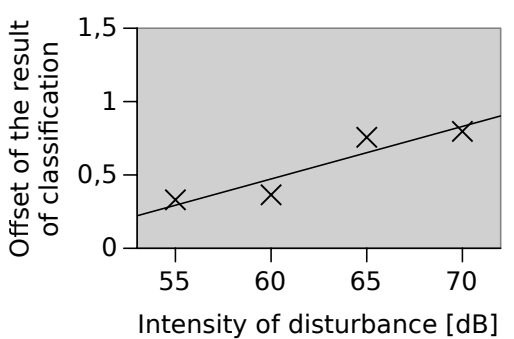

(e) Rock music

Fig. 5: Offset of the result of classification in function of intensity of disturbance 


\section{Analysis of all cases}

Assuming the case study as a model, an analysis of other fistulas sounds registered in dialysis station was performed. The only difference was that the disturbances were introduced artificially. The results obtained for each of the cases tested were consistent with the results obtained for the fistula registered in the anechoic chamber described in the previous section. It was assumed, therefore, that the model of artificial disturbance is correct and produces reliable results.

The results of all cases were averaged and shown in Tab.IV, and their graphical representation is shown in Fig.5. For the purposes of analysis, the letter labels $\boldsymbol{a}-\boldsymbol{f}$ were converted to numeric labels $\mathbf{1 - 6}$, which allowed for the calculation of the mean shift of the classification result relative to the base class.

TABLE IV: Offset of the result of classification as a result of disturbance

\begin{tabular}{c|ccccc}
$\begin{array}{c}\text { Intensity of } \\
\text { disturbance } \\
{[\mathrm{dB}]}\end{array}$ & $\begin{array}{c}\text { White } \\
\text { noise }\end{array}$ & $\begin{array}{c}\text { Urban } \\
\text { noise }\end{array}$ & $\begin{array}{c}\text { TV } \\
\text { mivie }\end{array}$ & Conversation & $\begin{array}{c}\text { Rock } \\
\text { music }\end{array}$ \\
\hline 55 & 0,11 & 0,51 & 0,18 & 0,44 & 0,33 \\
60 & 0,18 & 0,82 & 0,31 & 0,64 & 0,36 \\
65 & 0,70 & 1,11 & 0,52 & 0,82 & 0,76 \\
70 & 0,89 & 1,41 & 0,61 & 1,05 & 0,80
\end{tabular}

The increase in disturbance intensity in each of the cases studied led to an offset in the classification result, with the algorithm reporting worse condition of the fistula than what it actually was. It seems that the value of this offset can be approximated as linearly dependent on the intensity of the disturbance. If that is correct, extrapolating the trend line for smaller than tested noise intensities, it can be concluded that in order to obtain a sound quality sufficient for correct classification, the level of ambient noise should not exceed approximately $45 \mathrm{~dB}$.

\section{CONCLUSION}

The extraction algorithm proved to be quite resistant to disturbance - even loud noises at $70 \mathrm{~dB}$ did not prevent it from working, reducing only the amount of correctly recognized heart beats by $25 \%$. However, robustness of the extraction algorithm has not translated into maintaining the quality of the result of classification - noises of even slight intensity caused significant distortion of results.

A positive aspect of the study is the awareness that, if the recording is conducted under abusive acoustic conditions, the result will be unequivocally falsified in the pathology of the fistula. Its result will prompt the patient to verify rather than to optimistically accept misleading indication.
It is worth noting that only material recorded under low noise conditions allows for reasonable confidence in the results of classification process. While more complex denoising schemes (for example using a second microphone) seem possible, the complexity of implementing and testing such a solution makes it unfeasible.

\section{REFERENCES}

[1] Marcin Grochowina, Lucyna Leniowska and Piotr Dulkiewicz, "Application of Artificial Neural Networks for the Diagnosis of the Condition of the Arterio-venous Fistula on the Basis of Acoustic Signals," Brain Informatics and Health, Springer, 2014, pp. 400-411.

[2] Marcin Grochowina and Lucyna Leniowska, "Comparison of SVM and $\mathrm{k}-\mathrm{NN}$ classifiers in the estimation of the state of the arteriovenous fistula problem," Proceedings of the 2015 Federated Conference on Computer Science and Information Systems (FedCSIS), IEEE, 2015, pp. 249-254.

[3] Grochowina Marcin and Lucyna Leniowska, "The new method of the selection of features for the k-NN classifier in the arteriovenous fistula state estimation," Proceedings of the 2016 Computer Science and Information Systems (FedCSIS), IEEE, 2016, pp. 281-285.

[4] Grochowina Marcin and Lucyna Leniowska, "Analysis of the head prototype acoustic parameters for the acquisition of arteriovenous fistula signal," Mechanics in Medicine 12 (2014), University of Rzeszow, 2014, pp. 64-73. (in Polish)

[5] Mikkel Grama , Jens Tranholm Olesena , Hans Christian Riisa , Maiuri Selvaratnama and Michalina Urbaniaka, "Stenosis detection algorithm for screening of arteriovenous fistulae," 15th Nordic-Baltic Conference on Biomedical Engineering and Medical Physics (NBC 2011), Springer, 2011, pp. 241-244

[6] Duda Richard and Hart Peter and Stork David G "Pattern classification", John Wiley \& Sons 2012.

[7] Aha David and Kibler Dennis "Noise-Tolerant Instance-Based Learning Algorithms", IJCAI 1989, Citeseer, 1989, pp. 794-799,

[8] Jain Anil and Ross Arun "Learning user-specific parameters in a multibiometric system", Image Processing. 2002. Proceedings. 2002 International Conference on, IEEE, 2002, pp. I-I.

[9] Grochowina Marcin and Lucyna Leniowska, "The selection of features for the svm classifier in the arteriovenous fistula state estimation on the basis of acoustic signal", Acta Bio-Optica et Informatica Medica. Inżynieria Biomedyczna, vol.22(4), pp. 207-212. (in Polish)

[10] Domeniconi Carlotta and Peng Jing and Gunopulos Dimitrios "Locally adaptive metric nearest-neighbor classification", IEEE Transactions on Pattern Analysis and Machine Intelligence vol.1, IEEE, 2002, pp. 12811285.

[11] Aleksander Cisłak and Szymon Grabowski, "Experimental evaluation of selected tree structures for exact and approximate k-nearest neighbor classification," Proceedings of the 2014 Federated Conference on Computer Science and Information Systems, IEEE, 2014, pp. 93-100.

[12] Przemysław Wiktor Pardel and Jan G. Bazan and Jacek Zarychta and Stanisława Bazan-Socha, "A two-level classifier for automatic medical objects classification," Proceedings of the 2015 Federated Conference on Computer Science and Information Systems, IEEE, 2015, pp. 139-143.

[13] "WEKA documentation," http://www.cs.waikato.ac.nz/ml/weka/ documentation.html

[14] "Audacity manual," http://manual.audacityteam.org/\#tutorials

[15] "Digital Sound Level Meter AZ8921," http://www.az-instrument.com. tw/az-instrument/en/productsinfo/147.html 